Original Research Paper

\title{
IPv6 Transition Mechanism on UKMNet Network Environment
}

\author{
${ }^{1}$ Mohd Azi Bin Abdullah and ${ }^{2}$ Nor Effendy Othman \\ ${ }^{I}$ Department of Network, Center for Information Technology, \\ National University of Malaysia, 43600 UKM Bangi Selangor, Malaysia \\ ${ }^{2}$ Research Center for Software Technology and Management (SOFTAM), \\ Faculty of Information Science and Technology, National University of Malaysia, 43600 UKM Bangi Selangor, Malaysia
}

Article history

Received: 07-03-2016

Revised: 28-07-2016

Accepted: 14-12-2016

Corresponding Author:

Mohd Azi Abdullah

Department of Network, Center

for Information Technology,

National Universiti of Malaysia

(UKM), Bangi Selangor,

Malaysia

Email: aziabdullah@ukm.edu.my

\begin{abstract}
The production of IPv4 has ceased its supply and this has created a global phenomenon that is faced by Internet providers and users around the world. This means that IPv6 implementation phase has begun and IPv4 duties will be taken over by the IPv6 protocol. Technically, IPv4 and IPv6 cannot communicate directly due to the difference of architecture. IPv6 transition mechanism is used as a medium to allow both protocols to communicate and exist together on the same network. This study is performed to examine and investigate the performance of the 6to 4 and Dual Stack transition mechanism based on performance metrics like throughput, jitter and end-to-end delay when implemented on UKMNet network. The test infrastructure is built on the UKMNet network and the test was conducted using the testbed approached. The final result found that the 6to 4 mechanism is more reliable and easy to implement on UKMNet environment compared to dual stack mechanism.
\end{abstract}

Keywords: 6to4, Dual Stack, Testbed, Transition Mechanism, Throughput, Jitter, End-to-End Delay, TCP

\section{Introduction}

The deterioration of IPv4 addresses has led Internet Engineering Task Force (IETF) to create Internet Protocol next generation (IPng) or IPv6. IPv4 address deterioration issue was first detected in August 1990 by a group of researchers. Goth (2012) in his report stated On February 3, 2011, Internet Assigned Numbers Authority (IANA), an organization responsible for managing the global IPv4 has distributed the last blocks of IPv4 addresses to the Regional Internet Registry (RIR) and this is the sign that IPv4 production is over. Asia Pacific Network Information Center (APNIC), the RIRs distribute IP addresses to Malaysia has also made the final distribution of their IP addresses in the same year, on April 19, 2011 and this means that the IPv6 transition in Malaysia should have entered implementation phases. This phenomenon indirectly creates a scenario of coexistence between IPv4 and IPv6 protocols.

IPv6 protocol is designed to cover vulnerabilities found in IPv4. It was developed to ensure the continuation of IP addressing. Rafiee et al. (2012) stated in his research that the main advantages of IPv6 are it huge address space feature. According to Lee and Lough (1998), IPv4 and IPv6 protocol aren't able to communicate directly due to the architecture that is significantly different. To enable these protocols to communicate within the same network, an intermediary medium is needed to enable communication between both parties. An independent body, namely Internet world Internet Engineering Task Force (IETF) has developed IPv6 transition mechanisms. This mechanism is designed as an intermediate medium for IPv4 and IPv6 protocol to communicate.

The IPv6 transition cannot be implemented overnight. It needs to be well planned before commencing the implementation phase. According to $\mathrm{Wu}$ et al. (2013) transition process needs to be conducted phases by phase due to operating costs, utilities and change factors that should be considered by an organization before commencing the transition process. Therefore, the selection of suitable IPv6 transition mechanisms can ensure the transition process goes smoothly. Lu (2011) in his paper mention that the operation of campus network is managing a mass 
number of IP addresses, fast data transmitting, with high definition image and security management. Wang and $\mathrm{Xu}$ (2011) added that proper testing procedures are the crucial requirement in order to test the IPv6 transition mechanism before implementing on the actual network. UKMNet is a campus network for National University of Malaysia (UKM). Yousafzai et al. (2014) in his research stated that the development of IPv6 networks in UKM is still at the minimum rates. The aim of this study was to test the suitability of the IPv6 transition mechanisms based on existing UKMNet network specifications. Abdullah and Othman (2015) have reviewed the current studies from 2009 to 2015 of IPv6 transition performance on testbed setup.

In this study, two IPv6 transition mechanisms have been tested on testbed network that was built on UKMNet campus network. The focus of this paper is intended to assist the implementation of IPv6 transition mechanisms on the campus network and to investigate the performance of the transition mechanism before commencing the actual implementation phases. The organization of this paper is as follows: Section II contains a brief introduction of dual stack and tunneling mechanism. Section III shows the brief description of the testbed setup. Section IV contains the evaluation of the test performed. Section V contains analysis and the final result of the experiment. The end of the section is the conclusion of the study.

\section{Transition Mechanism}

\section{Dual Stack Mechanism}

Dual stack mechanism is the main mechanism between the three mainstream types of IPv6 transition mechanisms. The objective of the mechanism is to enable the network device to communicate in two different network protocols simultaneously. This is to apply interoperability between IPv4 and IPv6 protocol during the initial phase of the IPv6 transition migration process. Xiaohong (2013) mentioned that Dual stack mechanism can be implemented on hardware only or to the entire network that requires two protocols to operate simultaneously. This means that there will be a two different protocol stack within the network device. If the communication were initiated using the IPv6 protocol, the IPv6 datagram must travel via IPv4 network to reach its destinations. Datagram header is analyzed to determine the destination of packets sent. In addition, dual stack mechanism does not allow direct communication from IPv4 to IPv6 or vice versa. Therefore, Dual Stack mechanism will map the IPv6 datagram to the IPv4 datagram and send it via the IPv4 network.

According to Azcorra et al. (2010), Dual stack mechanism however requires double efforts in managing the routing and addressing. This could cause burden to the device that is running the Dual stack mechanism. Guan and Xia (2009) show the Dual stack mechanisms increase the burden on the network because it needs to do the routing process on two protocols at the same time. The operation of dual stack mechanism allows the IPv4 only node to communicate with other IPv4 only nodes when the IPv4 stack is enabled and vice versa. Hong (2014) in his study found that only one protocol could be running at a time. The Dual stack router needs to do the protocol stack binding process and this could increase routers burden and it efficiency will be decreased. Dual stack could not solve the IPv4 exhaustion problem because the mechanism itself still depends on IPv4 address.

\section{Tunneling Mechanism}

The tunneling mechanism uses tunnel concept to transport traffic from one location to another. In IPv6 transition mechanism cases, tunnel mechanism is used to connect two islands of same protocol via an ocean of different protocol. The idea is to connect two networks from the same protocol via a network from a different protocol.

According to Narayan and Tauch (2010) IPv6 datagrams is encapsulated into IPv4 packets before being sent via IPv4 network infrastructure to other IPv6 host on the network. For example in order to send IPv6 datagram via IPv4 network, the IPv6 datagram need to be encapsulated within the IPv4 datagram at the tunnel starting point so it can travel via the IPv4 network. When the encapsulated IPv6 datagram reaches the tunnel endpoint it will be de-capsulate so that the IPv6 datagram can be sent to its destination. Vermani (2012) in his study mention that there are two implementation types of tunnel mechanism, namely Automatic Tunneling and Configured Tunneling. Automatic tunneling mechanism does not need both endpoints to be configured but for manual tunneling one or both of the tunnel end points need to be configured. The 6to4 mechanism is one of the automatic tunneling mechanism. 6to4 enable the IPv6 network to connect to another IPv6 network via IPv4 network.

Taib and Budiarto (2010) mentioned in their study that Tunnel mechanism does not have a built in security features. The verification process is performed by checking the source address in the IPv4 packet header. This situation is causing problems where IP spoofing and packet injecting are major threat to the Tunneling mechanism. Saraj et al. (2014) added the two networks that are connected by Tunnel mechanism are unable to communicate directly and communication must go through an intermediate medium that can lead to decrease of reliability of subsequent data.

For further discussion on the current performance of IPv6 transition mechanism studies can be found on 
author other journal (Abdullah and Othman, 2015) that mainly focus on the review of IPv6 transition mechanism implementation on campus network.

\section{Testbed Setup}

In previous studies, Yousafzai et al. (2014) have built IPv6 test infrastructure on UKMNet campus network. This research is intended to evaluate the performance of 6to4 and Dual Stack mechanism based on TCP and UDP traffic perspective when implemented on UKMNet infrastructure. The parameter used to evaluate the network performance is based on QoS parameter that focuses only on data transmission on TCP traffic. According to Abdullah and Othman (2015), most of the researcher used throughput, jitter and end-to-end delay performance metric that were tested on TCP and UDP traffic to evaluate tunnel type and Dual Stack transition mechanism.

The test equipment will be installed on the real UKMNet campus network and the simulated traffic will be transmitted on the VLAN created for the test purpose.
The parameters used on this testbed specification are based on current specification of UKMNet. This is to imitate the current scenario of the UKMNet environment. Figure 1 shows the general overview of the testbed setup on the UKMNet environment.

\section{The Setup}

The testbed setup contains router, workstation and a server. This device was installed and attached to the existing UKMNet network device. Here both workstation and server were attached to the different part of the network. Router were used to route the simulation traffic to the other endpoint of the test infrastructure. Both of the transition mechanisms tested were configured on the end-to-end router. DITG software was installed on both endpoints to generate simulation for the network traffic. At the end of the network, Wireshark software is used to collect and analyzed the network traffic. Table 1 shows the specification of the testbed setup and the parameter used during the test.

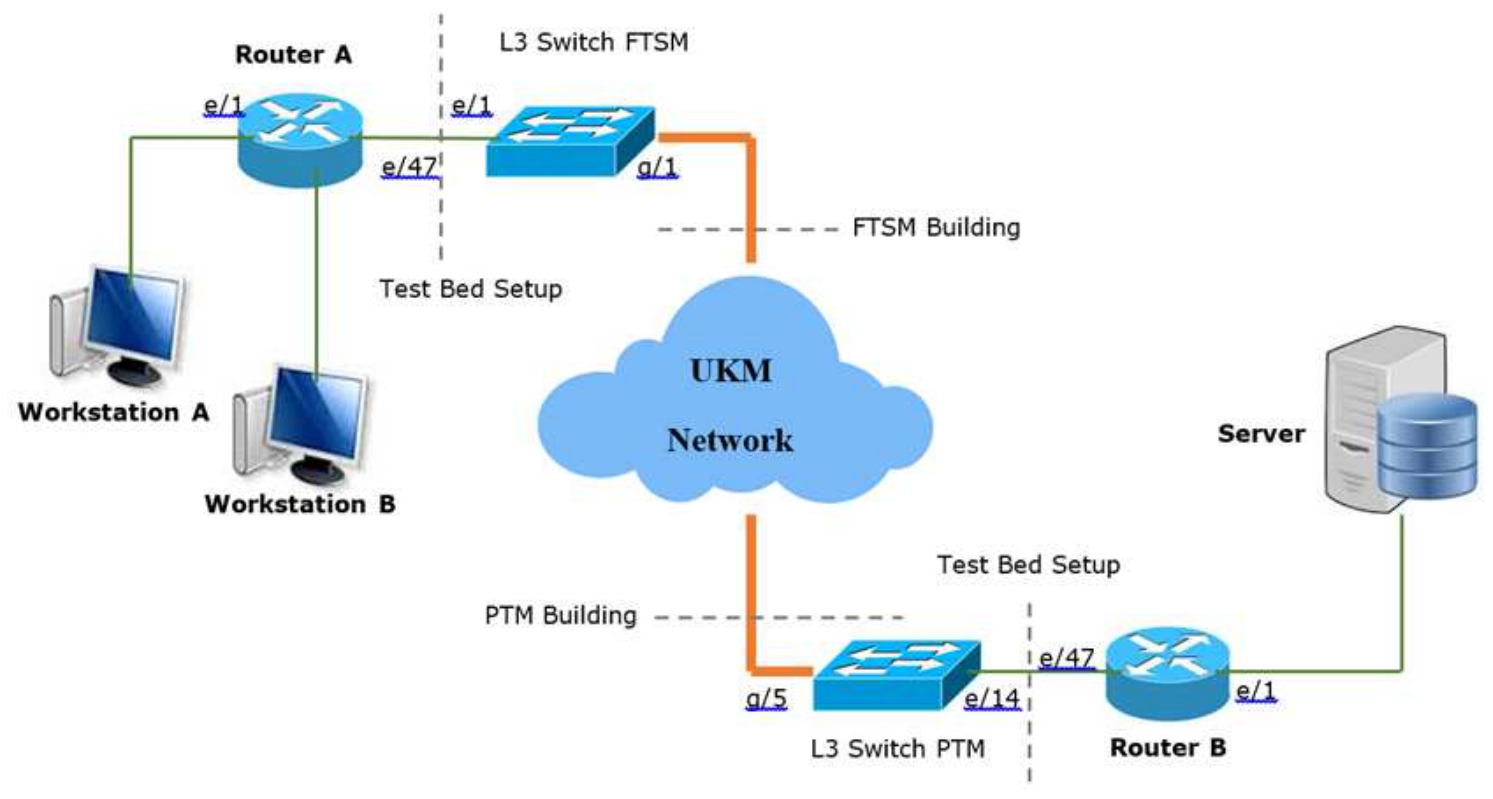

Fig. 1. Testbed setup

Table 1. Testbed Specification

\begin{tabular}{ll}
\hline Parameter & Specification \\
\hline Server Operating System & Windows Server 2008 R2, Intel Xeon Quad Core \\
Workstation Operating System & Windows 7, Intel Pentium 4.641/3.2 Ghz \\
Packet Size & 1500 byte \\
Duration & 5 minute \\
Performance Metrics & Throughput, Jitter, End-to-End Delay \\
Traffic used & TCP and UDP \\
Mechanism & 6to4 and Dual Stack \\
\hline
\end{tabular}




\section{Evaluation}

\section{Performance Metrics}

According to the study made by Abdullah and Othman (2015), most of the IPv6 researchers often use Quality of Service (QoS) parameter like throughput, jitter and end-to-end delay to determine their network performance when implemented with IPv6 transition mechanism. Some of the researcher like Aazam et al. (2011; Bahaman et al., 2012; Hadiya et al., 2013; Narayan and Tauch, 2010) prefer testbed approach to measure network performance compared to simulation approach when implemented with IPv6 transition mechanism. Throughput in network perspective can be elaborate as the number of data that can be transmitted from one location to another. These parameters are used to measure workload on the network. Throughput parameter enables the measurement on how many packets that can be processed by the network based on the time given. Jitter is the difference between the time taken for a packet to reach its destination. Jitter usually does not cause interference on the network, but it can be used to evaluate transmission performance using UDP datagram transmission in a network. Jitter parameters used to test the workload router. The effectiveness of data transmission on a network is dependent on the router used. The end-to-end delay is defined as the time taken by the packet data transmitted from the source node to the destination node across the network. End-toend delay parameters are calculated by subtracting the packet arrives at the destination node with the packets sent by the source node. The difference of the time is known as the end-to-end delay. This parameter is used to test the ability of a router in data transmission. Figure 2 and 3 show the 6to4 and Dual Stack testbed setup on UKMNet network.

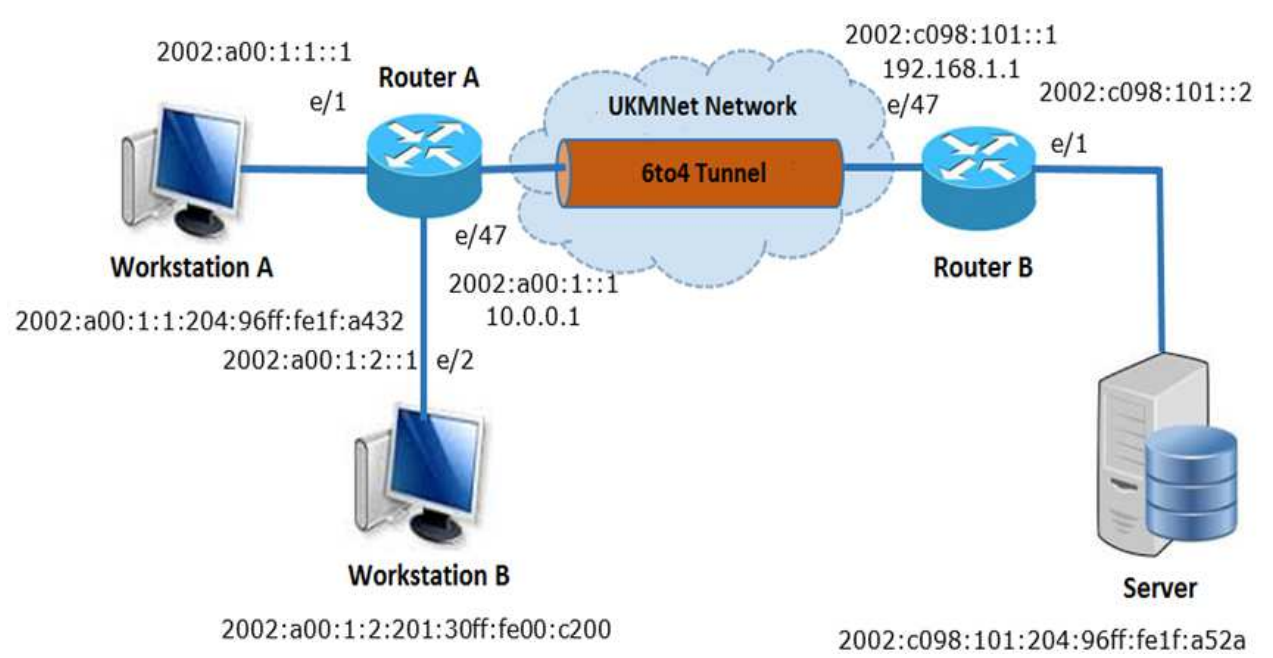

Fig. 2. 6to4 testbed setup

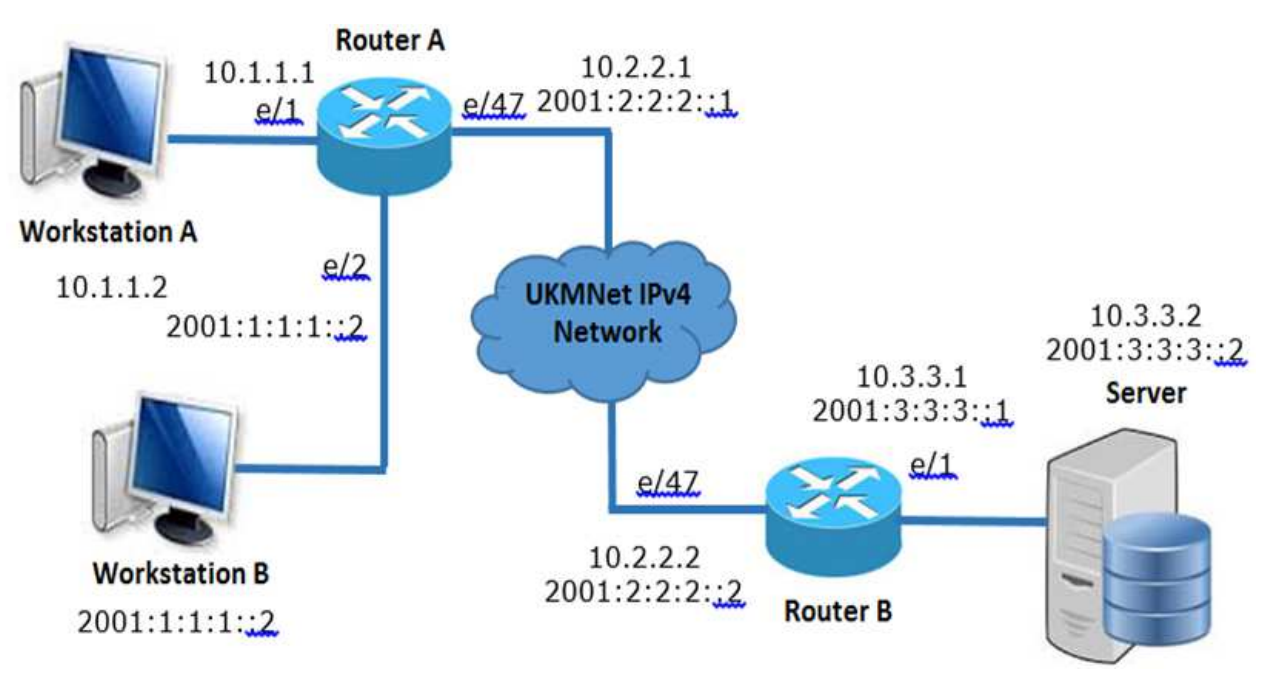

Fig. 3. Dual stack testbed setup 


\section{Result and Analysis}

\section{Result}

The metrics used to evaluate the performance of the network are throughput, jitter and end-to-end delay. Table 2 summarizes the result of comparison between Dual Stack mechanism and 6to4 mechanism in our experiment. The result shows that 6 to 4 mechanism achieved higher throughput, lower UDP jitter and TCP end-to-end delay than Dual Stack mechanism. The next sub section will further describe and analyze the result gained from the experiment.

\section{Analysis}

Based on Fig. 4, throughput for 6 to 4 mechanism shows better result compared to the Dual Stack throughput. The average 6to4 mechanism throughput value is $471 \mathrm{kbps}$ and the average value of Dual Stack mechanism throughput is $279 \mathrm{kbps}$. This means 6 to 4 mechanism can process within 37-40 packets per second and Dual Stack mechanisms can only process 25-30 packets per second. The throughput for Dual Stack mechanisms is lower than 6to4 mechanism is due to the burden borne by equipment that adapts the
Dual Stack mechanism. Dual Stack mechanism has to process two protocols simultaneously and this leads to increase of processor burden and workload. The cost also will increase due to the need of high-end router product to accommodate the needs of high power processing to ensure the smooth network operation. Figure 5 shows the average jitter for the 6to 4 mechanism is 0.009 milliseconds while the average jitter for the mechanism Dual Stack is 1.376 milliseconds. The difference in the average jitter for both of these mechanisms is of 1.367 milliseconds. 6to4 mechanism jitter value is lower than the Dual Stack mechanism due to router workload that processes the packet is low and the router can process and release the packet into the network at the rapid pace. Figure 6 graph shows the average value of endto- end delay tested on TCP traffic for the 6to4 mechanism is 1,223 milliseconds while the mechanism Dual Stack is 1.314 milliseconds. Delay time difference between the two mechanisms is 0.009 milliseconds per packet. This shows the mechanism Dual Stack has a slight time delay mechanisms than 6to4. The difference proof the processing router for Dual Stack mechanism is higher than 6 to 4 mechanism.

Table 2. Test Result

\begin{tabular}{llr}
\hline & Mechanism & \\
Metrics & ---------- & to4 \\
\hline TCP Throughput (kbps) & Dual stack & 471.000 \\
UDP Jitter (ms) & 279.000 & 0.009 \\
TCP End-to-End Delay (ms) & 1.376 & 1.223 \\
\hline
\end{tabular}

TCP Throughput on UKMNet Network

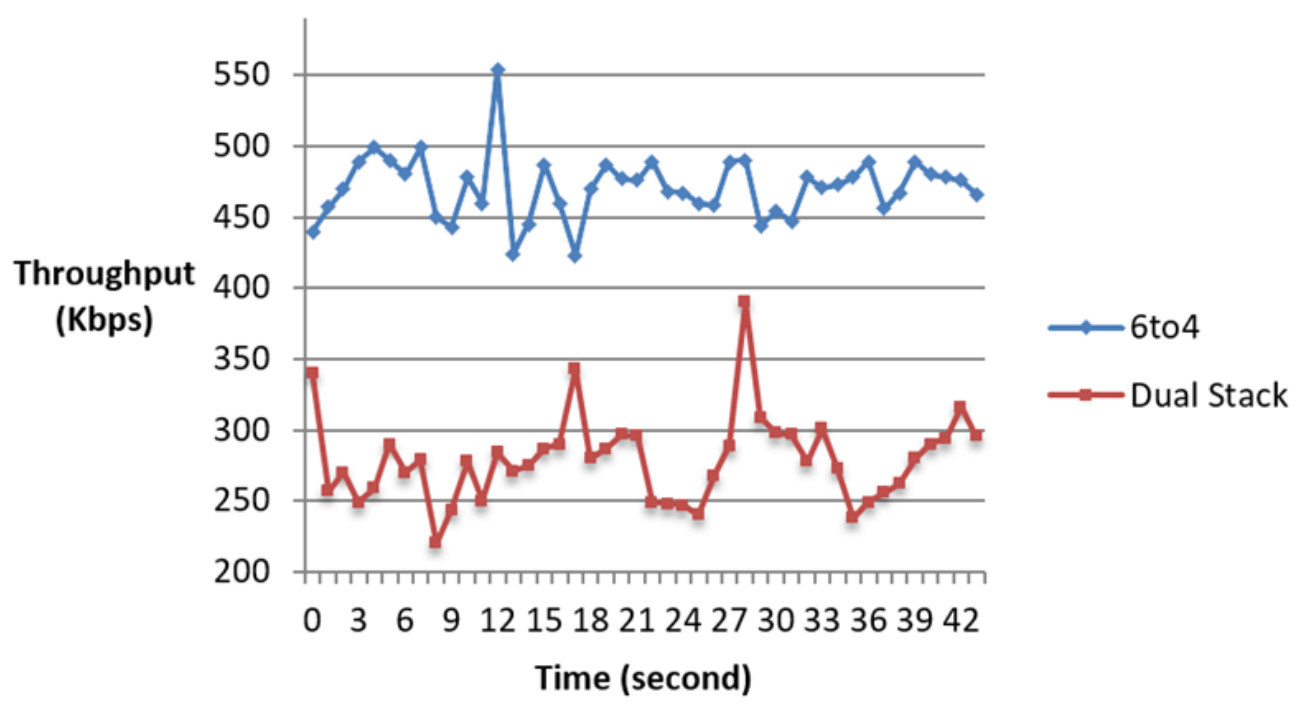

Fig. 4. Throughput graph 


\section{UDP Jitter On UKMNet Network}

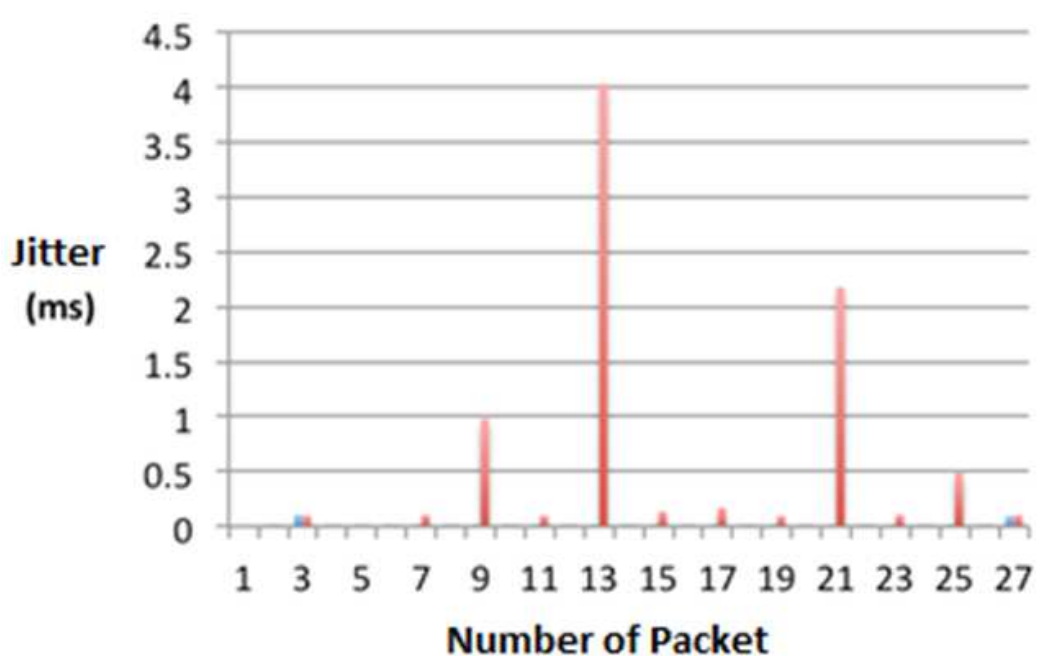

= 6 to 4

Dual Stack

Fig. 5. Jitter Graph

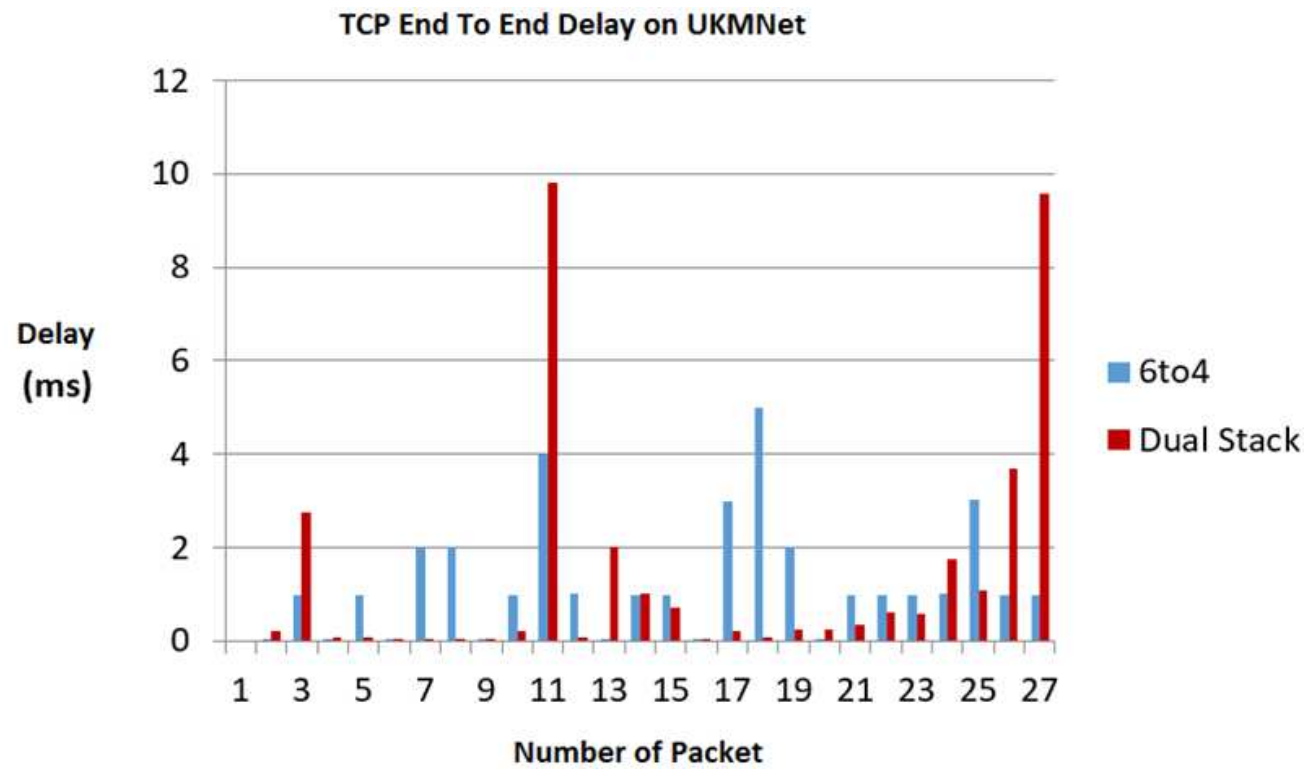

Fig. 6. End-to-end delay graph

In contrast to the Dual Stack mechanism, 6to4 mechanism performs encapsulation process on the tunnel start point and de-capsulate the datagram at the tunnel end point. The IPv6 datagram will be encapsulated into the IPv4 datagram and will be sent via the IPv4 network. When the datagram reached the tunnel end point, it will be de-capsulate and the IPv6 datagram will be sent to its destination. The encapsulation and de-capsulation process involves only on the router part and does not involve any other equipment.

\section{Conclusion}

IPv6 has become a necessity due to the deterioration of IPv4 addresses. This is to accommodate the growing number of users emerging technology. The migration from IPv4 to IPv6 protocol requires a long period of time to be implemented. This process requires IPv4 and IPv6 to coexist in one network and IPv6 transition mechanism is needed to enable both protocols to communicate. A proper planning and suitable selection of transition 
mechanism can avoid any disruption to the current network during the migration phase.

This study is performed to find the best transition mechanism to be implemented on UKMNet network and all the specification build in this study is based on UKMNet network environment. Overall the test results found that the 6 to 4 mechanisms tunnel type is more suitable to be implemented on the UKMNet compare to Dual Stack mechanisms. This is due to the ease and simple configuration compared to Dual Stack mechanisms that are complex and costly to be applied to the networks. The test also proved that network equipment that implements Dual Stack mechanisms have a high workload compare to equipment that implement with the 6 to 4 mechanism.

\section{Funding Information}

This work was funded by National University of Malaysia (UKM) under grant no. GGPM-2015-005.

\section{Author's Contributions}

All authors equally contributed in this work.

\section{Ethics}

This article is original and contains unpublished material. The corresponding author confirms that all of the other author have read and approved the manuscript and no ethical issues involved.

\section{References}

Aazam, M., A.M. Syed, S.A.H. Shah, I. Kham and M. Alam, 2011. Evaluation of 6to4 and ISATAP on a test LAN. Proceedings of the IEEE Symposium on Computers and Informatics, Mar. 20-23, IEEE Xplore Press, pp: 46-50. DOI: 10.1109/ISCI.2011.5958881

Abdullah, M.A. and N.E. Othman, 2015. Review of IPv6 transition mechanism implementation on campus network. Int. J. Commun. Antenna Propagat., 5: 269-278. DOI: 10.15866/irecap.v5i5.7086

Azcorra, A., M. Kryczka and A.G. Martinez, 2010. Integrated routing and addressing for improved IPv4 and IPv6 coexistence. IEEE Commun. Lett., 14: 477-479. DOI: 10.1109/LCOMM.2010.05.092449

Bahaman, N.A., E. Hamid and A.S. Prabuwono, 2012. Network performance evaluation of 6 to 4 tunneling. Proceedings of the International Conference on Innovation Management and Technology Research, Mar. 21-22, IEEE Xplore Press, pp: 263-268.

DOI: 10.1109/ICIMTR.2012.6236400
Goth, G., 2012. The end of IPv4 is nearly here really. IEEE Internet Comput., 16: 7-11. DOI: 10.1109/MIC.2012.37

Guan, T. and Y. Xia, 2009. The building of campus network transition scheme based on IPv6 automatic tunnel technology. Proceedings of the 2nd PacificAsia Conference on Web Mining and Web-based Application, Jun. 6-7, IEEE Xplore Press, pp: 109111. DOI: 10.1109/WMWA.2009.9

Hadiya, D., R. Save and G. Geetu, 2013. Network performance evaluation of 6 to 4 and configured tunnel transition mechanisms: An empirical test-bed analysis. Proceedings of the 6th International Conference on Emerging Trends in Engineering and Technology, Dec. 16-18, IEEE Xplore Press, pp: 5660. DOI: 10.1109/ICETET.2013.14

Hong, Z., 2014. Strategy and study of the transition technologies from IPv4 to TPv6. Proceedings of the International Conference on Electronics, Computer and Applications, May 8-9, IEEE Xplore Press, pp: 460-462.

DOI: 10.1109/IWECA.2014.6845656

Lee, D.C. and D.L. Lough, 1998. The internet protocol version 6. IEEE Potentials, 17: 11-12. DOI: $10.1109 / 45.666638$

Lu, Y.Y., 2011. Transition from IPV4 to IPV6 network application: Take a case as an example. Proceeding of the International Conference on Intelligence Science and Information Engineering, Aug. 20-21, IEEE Xplore Press, pp: 65-68. DOI: $10.1109 /$ ISIE.2011.139

Narayan, S. and S. Tauch, 2010. Network performance evaluation of IPv4-v6 configured tunnel and 6to4 transition mechanisms on windows server operating systems. Proceedings of the International Conference on Computer Design and Applications, Jun. 25-27, IEEE Xplore Press, pp: 435-440. DOI: $10.1109 /$ ICCDA.2010.5540939

Rafiee, H., M. Lowis and C. Meinel, 2012. IPv6 deployment and spam challenges. IEEE Internet Comput., 16: 22-29. DOI: 10.1109/MIC.2012.97

Saraj, T., M. Yousaf and S. Akhbar, 2014. ISP ISP Independent Architecture (IIA) for IPv6 packet traversing and inter-connectivity over hybrid (IPv4/IPv6) internet. Proc. Comput. Sci., 32: 973978. DOI: 10.1016/j.procs.2014.05.520

Taib, A. and R. Budiarto, 2010. Securing tunnel endpoints for IPv6 transition in enterprise networks. Proceedings of the International Conference on Science and Social Research, Dec. 5-7, IEEE Xplore Press, pp: 1114-1119. DOI: $10.1109 /$ CSSR.2010.5773699

Vermani, N., 2012. Mechanisms of tunneling IPv6 in IPv4 networks. Int. J. Scientific Eng. Res., 3: 1-9. 
Wang, Z. and W. Xu, 2011. Exploration of IPv6 network construction on campus. Proceeding of 7 th International Conference on Wireless Communications, Networking and Mobile Computing, Sept. 23-25, IEEE Xplore Press, pp: 14. DOI: 10.1109/wicom.2011.6040642

Wu, P., Y. Cui, J. Wu, J. Liu and C. Metz, 2013. Transition from IPv4 to IPv6: A state-of-the-art survey. IEEE Commun. Surveys Tutorials, 15: 1407-1424.

DOI: 10.1109/SURV.2012.110112.00200

Xiaohong, L., 2013. The research of network transitional technology from IPv4 to IPv6. Proceedings of the 4th International Conference on Digital Manufacturing and Automation, Jun. 29-30, IEEE Xplore Press, pp: 1507-1509.

DOI: 10.1109/ICDMA.2013.361
Yousafzai, M.M., R. Hassan, N.E. Othman, S.S. Hasan and U. Ali, 2014. Performance evaluation of IPv6 test-bed network at UKM. Proceeding of the 4th International Conference on Engineering Technology and Technopreneuship, Aug. 27-29, IEEE Xplore Press, pp: 63-66. DOI: $10.1109 /$ ICE2T.2014.7006220 